\title{
From spindle checkpoint to cancer
}

\author{
Christoph Lengauer \& Zhenghe Wang
}

Truncating and missense mutations in BUB1B, encoding the mitotic spindle checkpoint protein BUBR1, have been identified in individuals with mosaic variegated aneuploidy. This finding supports the idea that there is a genetic basis for aneuploidy in cancers.

Most human cancer cells are aneuploid, meaning they have an aberrant number of chromosomes. Whether gene mutations are responsible for this aneuploidy is debated ${ }^{1}$. In yeast, mutations in several genes, including genes involved in chromosome segregation and regulation of the mitotic spindle checkpoint, can result in aneuploidy. In humans, two mitotic spindle checkpoint genes, $B U B 1$ and $B U B 1 B$ (encoding BUBR1) have been reported to be mutated infrequently in colorectal cancers with chromosomal instability and in other aneuploid tumor types ${ }^{2,3}$. But convincing functional studies that could establish a causal connection between $B U B 1 B$ mutations and chromosomal instability have been missing. On page 1159, Sandra Hanks and colleagues ${ }^{4}$ report hereditary mutations in $B U B 1 B$ in individuals with a rare recessive disease called mosaic variegated aneuploidy (MVA) and provide such an important piece of evidence.

\section{MVA syndrome}

In 1986, Vigfusson et al. ${ }^{5}$ observed substantial developmental delay and apparent mental retardation in a male pigtail macaque monkey. They found that $75.7 \%$ of his lymphocyte nuclei were trisomic, but with the supernumerary chromosome varying from cell to cell. Examination of cultured skin fibroblasts showed a similar unusual karyotype.

A few years later, several humans, including two pairs of siblings, were found to have a similar cytogenetic phenomenon. Cells of these individuals frequently form micronuclei and have abnormal numbers of several chromosomes in multiple tissues, called mosaic aneuploidies (Fig. 1). For example, in 1991, the case of a 17-year-old girl with severe microcephaly and mental retardation was reported $^{6}$. Karyotype analyses of her phytohemagglutinin-stimulated lymphocytes, cultured skin fibroblasts, direct and cultured bone marrow and Epstein-Barr virus-trans-

Christoph Lengauer and Zhenghe Wang are at the Sidney Kimmel Comprehensive Cancer Center, Johns Hopkins University School of Medicine, Baltimore, Maryland 21231, USA. e-mail:lengauer@jhmi.edu

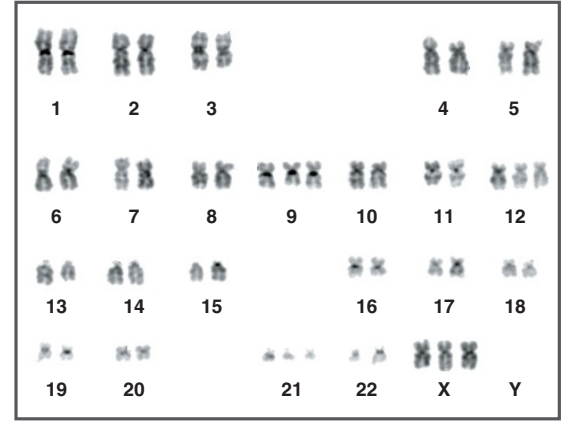

Figure 1 Karyotype of a cell from an individual with MVA. Instead of two copies (normal for a diploid cell), there are three copies each of chromosomes 9, 12, 21 and $X$.

formed lymphoblasts all showed that at least $10 \%$ of cells were trisomic with respect to many different chromosomes. Trisomies involving all chromosomes except chromsomes 5, 10, 13, 14 and 17 were observed. The existence of this mosaic trisomy in four different tissues and in repeated cultures over a 3-y period suggested that it was due to a genetic abnormality resulting in mitotic instability.

So far, only 15 cases of MVA have been reported worldwide ${ }^{7}$. It is not clear whether all cases represent the same condition, as some clinical and cytogenetic differences exist among them. For example, mitoses of some of the individuals have a higher frequency of separated centromeres and splayed chromatids in the presence of colcemid, a cytogenetic phenomenon referred to as premature centromere division ${ }^{8}$. The two most common clinical abnormalities of individuals with MVA are microcephaly and intrauterine growth retardation. Also, the risk of malignancy for the affected individuals is high. Almost one-third of the young affected individuals reported developed leukemia, rhabdomyosarcoma or Wilms tumor ${ }^{7}$.

\section{Aneuploidy and mitotic spindle checkpoint}

Matsuura et al. ${ }^{9}$ recently reported that skin fibroblasts cultured from six individuals with both premature centromere division and MVA showed a defect in the mitotic spindle checkpoint. This finding was not unexpected, as others had previously shown that deficiency of the spindle checkpoint components resulted in unequal segregation of chromosomes into daughter cells and thereby led to aneuploidy ${ }^{2}$. The mitotic checkpoint serves as a surveillance mechanism that ensures the faithful transmission of chromosomes from a mother cell to its two daughter cells during mitosis. It warrants that all chromosomes congress to the metaphase plate before separating into the two newly forming daughter cells, and that the chromosome kinetochores attach properly to the spindle microtubules. Many important members of this checkpoint have been identified, including the genes Bub1, Bub3, Mad1l1, Mad2l1, Mxd3 and Mps1 (ref. 10). Most of these regulatory components seem to function as inhibitors of a multisubunit E3 ubiquitin ligase, known as the anaphase promoting complex or cyclosome (APC/C), which presumably controls the exit from metaphase.

Mutational analysis of one of the mitotic checkpoint genes, $B U B 1 B$, in eight families with MVA by Hanks et al. ${ }^{4}$ showed that five families carry mutations in $B U B 1 B$ in both alleles. Four families have either nonsense or frameshift mutations in one allele that result in the premature truncation of the encoded BUBR1 protein, deleting its kinase domain and thus inactivating its function. The reported mutations of the second allele were missense mutations. Five (of six) missense mutations were located in the kinase domain of BUBR1. They probably also result in a loss of function, although functional studies are required to prove the case.

The paper by Hanks et al. ${ }^{4}$ is a perfect illustration of the idea that uncovering the underlying genetic basis of a rare disease with increased cancer risk can contribute to the understanding of general aspects of tumorigenesis.

\section{Chromosomal instability and cancer}

Like the supernumerary chromosomes that vary from cell to cell in individuals with MVA, most human cancers are aneuploid, and chromosomal instability presumably 
drives tumor progression by accelerating the rate of gain or loss of individual chromosomes. The molecular mechanisms of chromosomal instability in human cancers largely remain to be elucidated.

Several years ago, we hypothesized that mutations in human spindle checkpoint genes could cause chromosomal instability in cancers $^{11}$. We cloned the two human homologs of yeast $B u b 1$, called $B U B 1$ and $B U B 1 B$, and found that the two genes were mutated, albeit rarely, in chromosomal instability tumors of individuals with colorectal cancer ${ }^{2}$. This study provided the first evidence that gene mutations can result in a defective spindle checkpoint in humans and thus might be responsible for aneuploidy in human cancers. One $B U B 1 B$ mutation in an aneuploid colorectal cancer was a somatic deletion that was predicted to remove part of the protein's kinase domainanalogous to the truncating mutations now found in individuals with MVA.

Mutant mice that express low levels of BubR1 protein develop progressive aneuploidy but form tumors only after being challenged with a carcinogen ${ }^{12}$. These findings, as well as the low frequency of $B U B 1$ and $B U B 1 B$ mutations in cancers and the lack of gene mutations found in any other members of the mitotic checkpoint machinery thus far, led to questions regarding the importance and applicability of our original observations. The belief that spindle checkpoint defects cause aneuploidy in human tumors, however, is revived by pinning down $B U B 1 B$ mutations as the underlying genetic alteration in MVA.
1. Jallepalli, P.V. \& Lengauer, C. Nat. Rev. Cancer 1, 109-117 (2001).

2. Cahill, D.P. et al. Nature 392, 300-303 (1998).

3. Gemma, A. et al. Genes Chromosomes Cancer 29, 213-218 (2000).

4. Hanks, S. et al. Nat. Genet. 36, 1159-1161 (2004).

5. Vigfusson, N.V. et al. Cytogenet. Cell Genet. 42, 154-158 (1986)

6. Warburton, D., Anyane-Yeboa, K., Taterka, P., Yu, C.Y. \& Olsen, D. Ann. Genet. 34, 287-292 (1991).

7. Jacquemont, S., Boceno, M., Rival, J.M., Mechinaud, F. \& David, A. Am. J. Med. Genet. 109, 17-21 (2002).

8. Plaja, A. et al. Am. J. Med. Genet. 98, 216-223 (2001).

9. Matsuura, S. et al. Am. J. Hum. Genet. 67, 483-486 (2000)

10. Musacchio, A. \& Hardwick, K.G. Nat. Rev. Mol. Cell. Biol. 3, 731-741 (2002).

11. Lengauer, C., Kinzler, K.W. \& Vogelstein, B. Nature 396, 643-649 (1998).

12. Dai, W. et al. Cancer Res. 64, 440-445 (2004).

\title{
Complementing complexity
}

\author{
Trudy F C Mackay
}

One challenge in modern biology is to understand the detailed genetic basis of variation for quantitative traits, including complex behaviors. A new study shows that historical recombination in outbred strains combined with functional complementation tests can identify pleiotropic genes with small effects on naturally occurring variation for anxiety-related behaviors in mice.

\begin{abstract}
Most variation between individuals in behavior, physiology, morphology, disease susceptibility and reproductive fitness can be attributed to the segregation of multiple quantitative trait loci (QTLs) with individually small effects, whose expression is conditional on the environment ${ }^{1}$. Understanding the genetic and environmental factors that cause this variation is of fundamental importance for medicine, agriculture, evolution and the emerging discipline of functional genomics. Genome scans for QTLs have become a cottage industry in the past 15 years, facilitated by the discovery of abundant, highly polymorphic molecular markers in many species and the development of sophisticated statistical methods of analy$\mathrm{sis}^{2,3}$. Identifying the genes underlying QTL peaks by positional cloning has proven elusive in all but a handful of cases where the QTLs had larger than usual effects ${ }^{4}$. On page 1197

Trudy F. C. Mackay is in the Department of Genetics, Campus Box 7614, North Carolina State University, Raleigh, North Carolina 27612, USA.

e-mail:trudy_mackay@ncsu.edu
\end{abstract}

of this issue, Binnaz Yalcin and colleagues ${ }^{5}$ describe a combined genetic and functional strategy for identifying genes corresponding to QTLs with small effects.

\section{The elusive QTLs}

There are three main problems facing highresolution QTL mapping. First, an increasingly large number of recombination events in each QTL interval are required to map the QTLs to decreasingly smaller intervals. Second, individual QTLs have small effects that are sensitive to the environment; there-

\section{SNP 1 QTL SNP 2 Strain EMO}

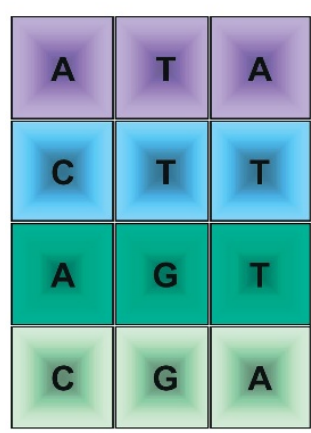

fore, the phenotype of a single individual is not a reliable indicator of QTL genotype. Third, even after whittling down the QTL interval to a region containing several positional candidate genes, one must determine which of them corresponds to the QTL. Guessing (the 'candidate gene' approach) has worked in cases where the genetic basis underlying the trait phenotype is well understood. But the availability of complete genome sequences for many organisms means it is possible to peruse the gene list in a candidate QTL region, inevitably finding many predicted 